\title{
Dermoscopic profile of pigmented purpuric dermatosis: new observations
}

\author{
Mahmut Sami Metin'1, Ömer Faruk Elmas² \\ ${ }^{1}$ Department of Dermatology and Venereology, Batman Medical Park Hospital, Batman, Turkey \\ ${ }^{2}$ Department of Dermatology and Venereology, Faculty of Medicine, Ahi Evran University, Kırşehir, Turkey \\ Adv Dermatol Allergol 2019; XXXVI (6): 687-691 \\ DOI: https://doi.org/10.5114/ada.2019.91419
}

\begin{abstract}
Introduction: Pigmented purpuric dermatosis (PPD) describes a cutaneous eruption that presents with asymptomatic or pruritic macules, and petechiae with red and brown pigmentation. Dermoscopy is a non-invasive diagnostic method making a more detailed examination possible and provides important clues to many dermatologic conditions. There are few original studies focusing on the dermoscopic aspect of PPD apart from case reports.

Aim: To identify dermoscopic findings of pigmented purpuric dermatosis, which will facilitate diagnosis by reducing the use of invasive procedures.

Material and methods: The study included the patients with histopathologically confirmed pigmented purpuric dermatosis. Demographic, clinical and dermoscopic features of all the cases were retrospectively reviewed.

Results: The study enrolled 15 (60\%) men and 10 (40\%) women. The mean age of the patients was 42 (age range: 28-72). The most common dermoscopic findings were red globules and red dots which were observed in all the cases $(100 \%)$, followed by coppery brown background (72\%), brown lines reticular (40\%) and subtle brown dots (40\%). The other findings were brown circles (32\%), red circles (32\%), grey dots (32\%), red background (8\%), serpentine vessels (8\%), rosette structures (8\%), thick brown lines (4\%), and thick linear vessels (4\%).

Conclusions: The characteristic dermoscopic findings of PPD have been defined in the relevant literature. Here we have identified some dermoscopic findings which have not been described previously: red circles, brown circles, rosette structures, light brown background and red background. Dermoscopic examination may facilitate the diagnostic process by reducing the use of invasive methods.
\end{abstract}

Key words: dermoscopy, pigmented purpuric dermatosis, Schamberg's disease.

\section{Introduction}

Pigmented purpuric dermatosis (PPD) describes a cutaneous eruption that presents with asymptomatic or pruritic macules, and petechiae with red and brown pigmentation. It mainly involves lower extremities and often has a chronic relapsing course. Etiopathogenesis of the disease is clearly unknown. Differential diagnosis includes bleeding diathesis, stasis dermatitis, cutaneous vasculitis, contact reactions, angioma serpiginosum and unilateral nevoid telangiectasia [1, 2].

Many different subtypes of PPD have been described. These subtypes include Schamberg's disease, lichen aureus, pigmented purpuric lichenoid dermatosis of Gougerot and Blum, purpura annularis telangiectoides (Majocchi's disease) and eczematid-like purpura of Doucas and Kapetanakis [1]. Schamberg's disease, also known as progressive pigmented purpuric dermatosis, is the most common form of PPD and typically shows a caudal-cranial progression of the red-brown pigmentation. Lichen aureus is an uncommon type of PPD and is clinically characterized by solitary or multiple rust macules, papules or plaques [3, 4].

The diagnosis of PPD is usually made clinically but, in some cases, histopathological examination may be needed to rule out similar conditions [5].

Dermoscopy is a non-invasive diagnostic method making more detailed examination possible and provides important clues to many dermatologic conditions. There are few original studies focusing on the dermoscopic aspect of PPD apart from case reports [6-9].

Address for correspondence: Ömer Faruk Elmas MD, Department of Dermatology and Venereology, Faculty of Medicine, Ahi Evran University, 40000 Kırşehir, Turkey, phone: +90 5330260679, e-mail: omerfarukmd@gmail.com Received: 24.11.2018, accepted: 30.01.2019. 


\section{Aim}

Here we have aimed to identify dermoscopic findings of PPD which will facilitate the diagnosis by reducing the use of invasive procedures.

\section{Material and methods}

The study included the patients with histopathologically confirmed pigmented purpuric dermatosis. All the patients were selected from the outpatient department of a tertiary centre between December 2017 and November 2018. Demographic, clinical and dermoscopic features of all the cases were retrospectively reviewed. Dermoscopic images of each lesion were obtained using a polarized handheld dermoscope (DermLite DL4; 3Gen Inc.; San Juan Capistrano, CA). Dermoscopic photographing was performed with a dermoscope adopted camera phone with a high resolution (iPhone 7 plus, Apple, California, USA).

\section{Statistical analysis}

The relationship between two categorical independent variables was evaluated using Chi square test. Descriptive statistics for numeric variables was represented as mean, and for categorical variables, as numbers and \% values. SPSS Windows version 24.0 package software (SPSS Inc.; Chicago, IL, U.S.A.) was used for statistical analysis.

\section{Ethical approval}

All the procedures followed were in accordance with the Helsinki Declaration and the study was approved by the local clinical research ethics committee.

\section{Results}

The study enrolled 15 (60\%) men and 10 (40\%) women. The mean age of the patients was 42 (age range: 28-72). The mean disease duration was 4 months (duration range: $1-48$ months). Nineteen (76\%) patients had Schamberg's disease and 6 (24\%) had lichen aureus. All the cases presented with bilateral pigmented patches located on lower extremities. The lumbar region was also affected in $3(12 \%)$ cases. The most common dermoscopic findings were red globules (100\%) and red dots (100\%), followed by coppery brown background (72\%), brown lines reticular (40\%) and subtle brown dots (40\%). All the dermoscopic findings observed are detailed in Table 1.

There were no statistically significant differences between the dermoscopic findings in relation to age, subtype of PPD or gender $(p>0.05)$.

\section{Discussion}

Pigmented purpuric dermatosis is a group of chronic skin diseases characterized by symmetric patchy pigmentation along with purpuric and petechial lesions. Superficial dermal perivascular lymphohistiocytic infiltration without leukocytoclastic vasculitis, marked hemosiderin deposition and erythrocyte extravasation are the main histological features of the disease; however, these histological findings are not specific to PPD and need clinical correlation [10].

Clinical subtypes of PPD include Schamberg's disease (SD), purpura annularis telangiectoides or Majocchi's disease (MD), lichen aureus (LA), pigmented purpuric lichenoid dermatosis, and eczematid-like purpura of Doucas and Kapetanakis. Their clinical presentation

Table 1. Dermoscopic findings of the cases with pigmented purpuric dermatosis

\begin{tabular}{|c|c|c|c|}
\hline Dermoscopic findings & $\begin{array}{l}\text { Schamberg's disease } \\
\qquad(N=19)\end{array}$ & $\begin{array}{l}\text { Lichen aureus } \\
\qquad(N=6)\end{array}$ & $\begin{array}{l}\text { Total number and percentage } \\
\qquad(N=25)\end{array}$ \\
\hline Red dots & $n=19 ; 100 \%$ & $n=6 ; 100 \%$ & $25(100 \%)$ \\
\hline Red globules & $n=19 ; 100 \%$ & $n=6 ; 100 \%$ & $25(100 \%)$ \\
\hline Coppery brown background & $n=13 ; 68 \%$ & $n=5 ; 83 \%$ & $18(72 \%)$ \\
\hline Light brown background & $n=8,42 \%$ & $n=2 ; 33 \%$ & $10(40 \%)$ \\
\hline Red background & $n=1 ; 5 \%$ & $n=1 ; 17 \%$ & $2(8 \%)$ \\
\hline Brown lines reticular & $n=8,42 \%$ & $n=2 ; 33 \%$ & $10(40 \%)$ \\
\hline Subtle brown dots & $n=7,37 \%$ & $n=3 ; 50 \%$ & $10(40 \%)$ \\
\hline Brown circles & $n=6 ; 32 \%$ & $n=2 ; 33 \%$ & $8(32 \%)$ \\
\hline Red circles & $n=6 ; 32 \%$ & $n=2 ; 33 \%$ & $8(32 \%)$ \\
\hline Subtle grey dots & $n=5 ; 26 \%$ & $n=3 ; 50 \%$ & $8(32 \%)$ \\
\hline Serpentine vessels & $n=1 ; 5 \%$ & $n=1 ; 17 \%$ & $2(8 \%)$ \\
\hline Thick brown lines & $n=1 ; 5 \%$ & - & $1(4 \%)$ \\
\hline Rosette structures & $n=1 ; 5 \%$ & $n=1 ; 17 \%$ & $2(8 \%)$ \\
\hline Thick linear vessels & $n=1 ; 5 \%$ & - & $1(4 \%)$ \\
\hline
\end{tabular}


enables distinguishing roughly between them; however, histopathological examination may also be needed [11].

In this study, the most common clinical form we observed was SD (76\%), followed by LA (24\%). The other forms of the disease were not observed in any patients. In a study of Cakmak et al. and a study of Ozkaya et al., the frequencies of the SD were $88.8 \%$ and $93 \%$, respectively $[6,7]$.

PPD is accepted to be more frequent in males than females [5, 9]. Similarly, our study also showed a male predominance. PPD can be seen in any age; however, the disease usually occurs in the $4^{\text {th }}$ and $5^{\text {th }}$ decades of life. In our study, similar to the previous studies, the median age was 42 years (range: 28-72) [12, 13].

Dermoscopy is a practical and non-invasive diagnostic tool used in many neoplastic and non-neoplastic dermatological conditions. Dermoscopic features of many dermatological conditions have been well described, however, there are few original studies investigating the dermoscopic aspect of PPD [6-9].

In the present study, the most common findings were red dots and red globules which were observed in all the cases (100\%) (Figures 1, 2). Similarly, in the study of Cakmak et al., all the lesions showed red dots and globules [6]. In the study of Ozkaya et al., red dots and globules were present in 69\% and $75 \%$ of the cases, respectively [7]. It has been suggested that the red dots and globules reflect extravasated red blood cells and an increased number of blood vessels [9].

Another frequent dermoscopic finding we observed was coppery brown background (72\%) (Figure 1). The frequencies of this finding were $100 \%$ and $97 \%$ in the studies of Cakmak et al. and Ozkaya et al., respectively $[6,7]$. Coppery brown background is thought to be associated with lymphohistiocytic dermal infiltration, extravasated erythrocytes and hemosiderin deposition [9]. We also observed light brown and red background in $10(40 \%)$ and 2 (8\%) cases, respectively (Figure 3). To the best of our knowledge, these colours of background have not been described for PPD previously. We suggest that the brown background is associated with increased epidermal melanin. The histological counterpart of the red background observed in PPD may be increased density of dermal vessels.

In the present study, brown lines reticular were observed in $40 \%$ of the cases (Figure 4). In the studies of Cakmak et al. and Ozkaya et al., the frequencies of this finding were $44.4 \%$ and $34 \%$, respectively $[2,5]$. The brown lines reticular reflect the presence of increased melanin in the epidermal rete pegs $[14,15]$.

Brown dots were present in $40 \%$ of the patients in our study (Figure 4). The frequencies of this finding were $16.6 \%$ and $53 \%$ in the studies of Ozkaya et al. and Cakmak et al., respectively $[6,7]$. Brown dots are thought to reflect spherical setting of pigmented melanocytes at the epidermal basal layer or melanophages in superficial dermis [14].

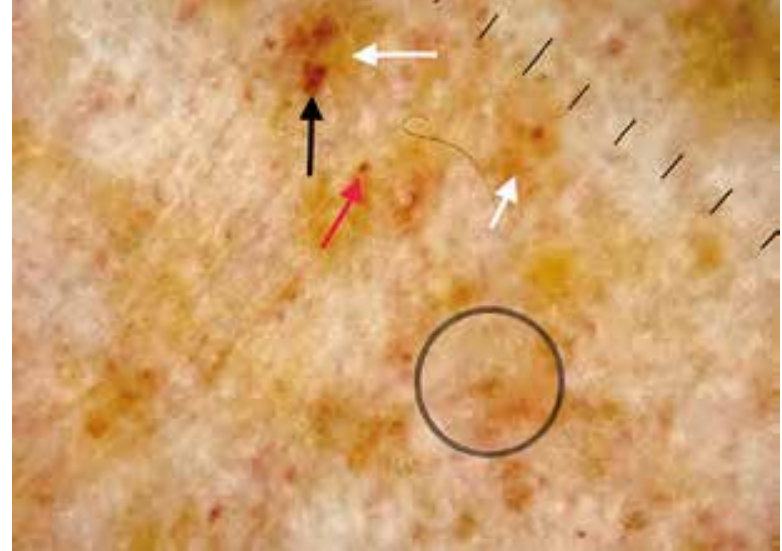

Figure 1. Red dot (red arrow), red globule (black arrow), coppery brown background (white arrows), brown circles (circle)

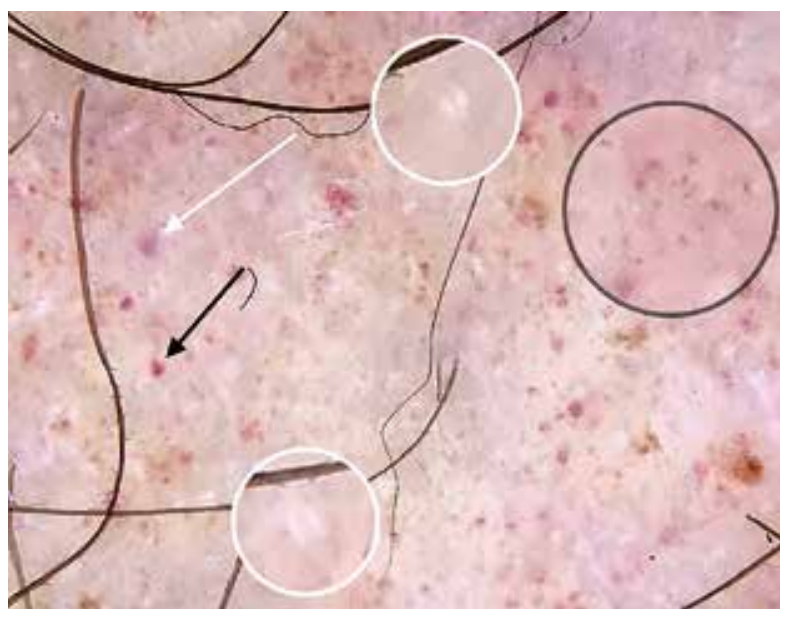

Figure 2. Red dot (black arrow), red globule (white arrow), rosette structures (white circles), subtle grey dots (black circles)

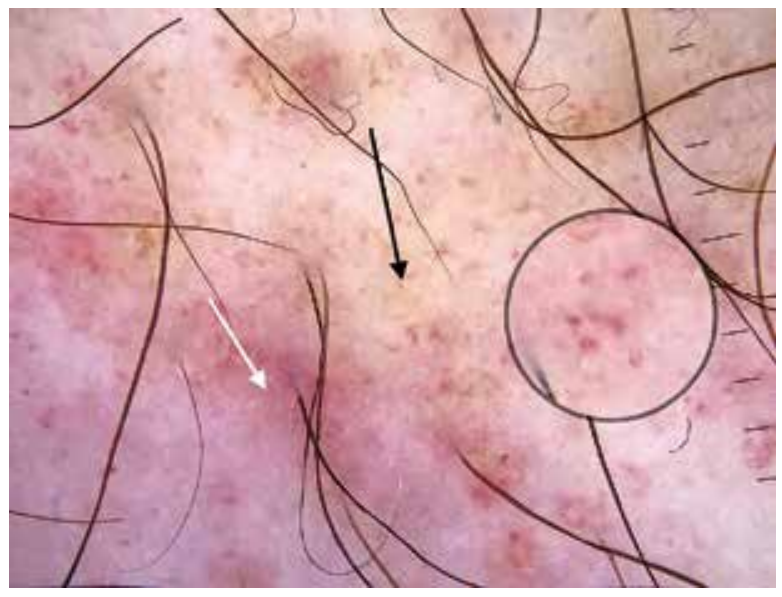

Figure 3. Red background (white arrow), light brown background (black arrow), subtle grey dots (grey arrows), red circles (black circles) 
We observed brown circles in $40 \%$ of the patients (Figure 1). Brown circles, to the best of our knowledge, have not been described for PPD previously. They are thought to be associated with partial pigmentation of rete ridges [16].

We observed red circles in 32 percent of the cases (Figure 5). We think that the red circles reflect dilated coiled vessels which can be visualized easily. To the best of our knowledge, they have not been described for PPD previ-

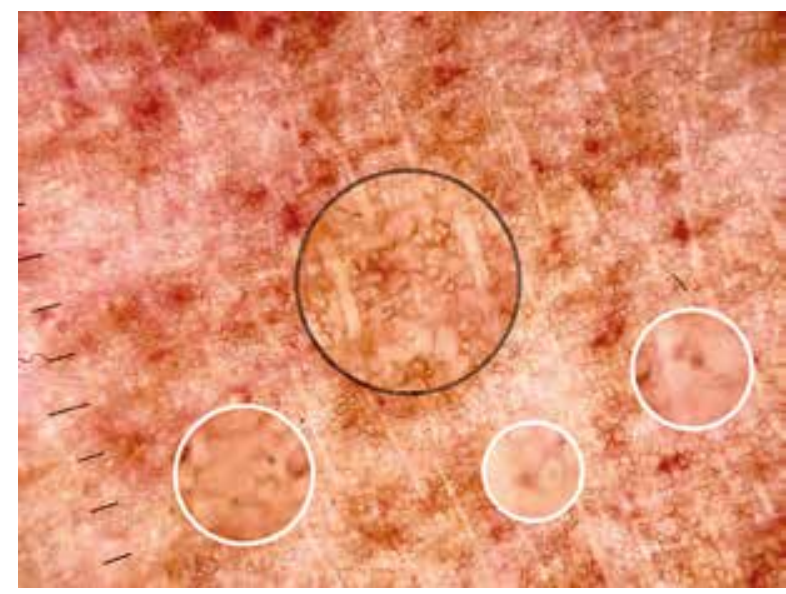

Figure 4. Brown dots (white arrows), brown lines reticular (circle)

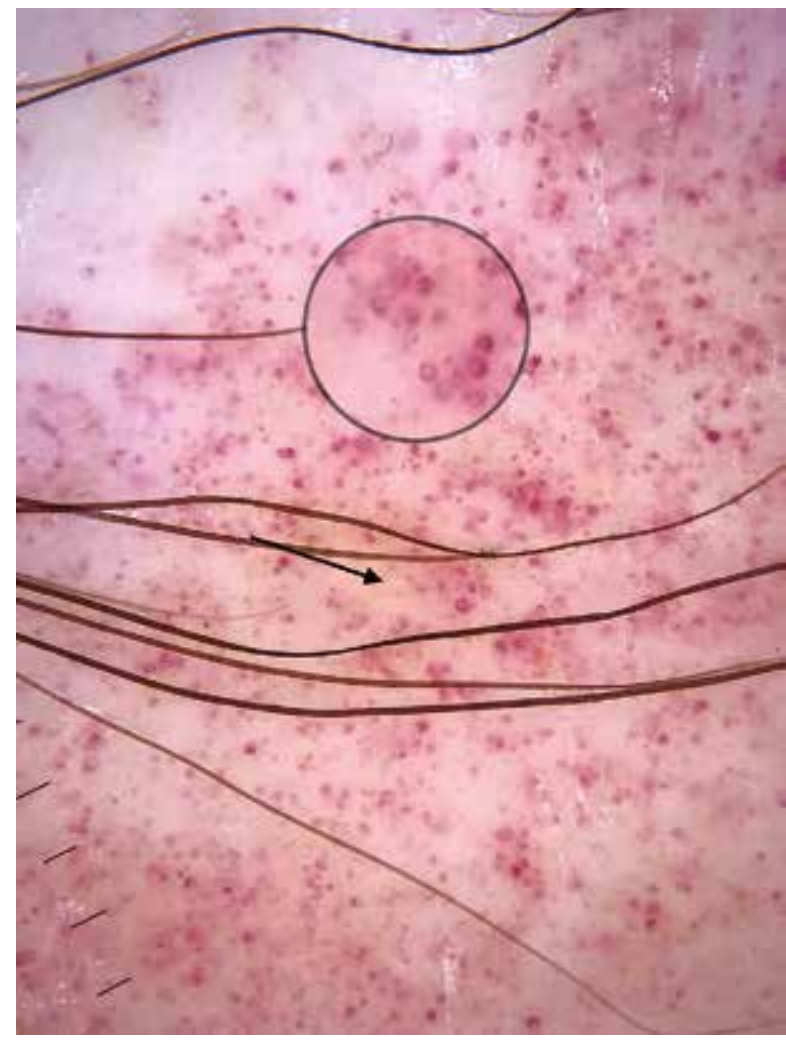

Figure 5. Red circles (circles), light brown background (arrow) ously. We also observed thick linear vessels and irregular fine linear vessels (serpentine vessels) in 1 and 2 cases, respectively. It should be kept in mind that presence of prominent vascular structures on the lower leg may also be associated with venous insufficiency or just the anatomic site itself. The main dermoscopic finding of stasis dermatitis is glomerular vessels [17]. None of the cases showed glomerular vessels in the present study.

Subtle grey dots were detected in 8 (32\%) cases (Figures 2,4$)$. The study of Cakmak et al. also showed the presence of grey dots in $3(16.6 \%)$ cases. The histological counterpart of grey dots is thought to be melanin pigment within the papillary dermis, either free, in nests of melanocytes or in melanophages [16].

We detected rosette structures in 2 (8\%) patients (Figures 2,6$)$. The histological counterpart of rosette structures is clearly unknown but is thought to be associated with the interaction of keratin-filled adnexal openings with the polarized light of the dermoscope. Rosette structures can be seen in many conditions including actinic keratosis, basal cell carcinoma, melanoma and squamous cell carcinoma [18]. To our knowledge, rosette structures have not been described previously for PPD.

Differential diagnosis of PPD includes bleeding diathesis, stasis dermatitis, cutaneous vasculitis, mycosis fungoides, contact reactions, angioma serpiginosum and unilateral nevoid telangiectasia [1, 2]. Dermoscopic exami-

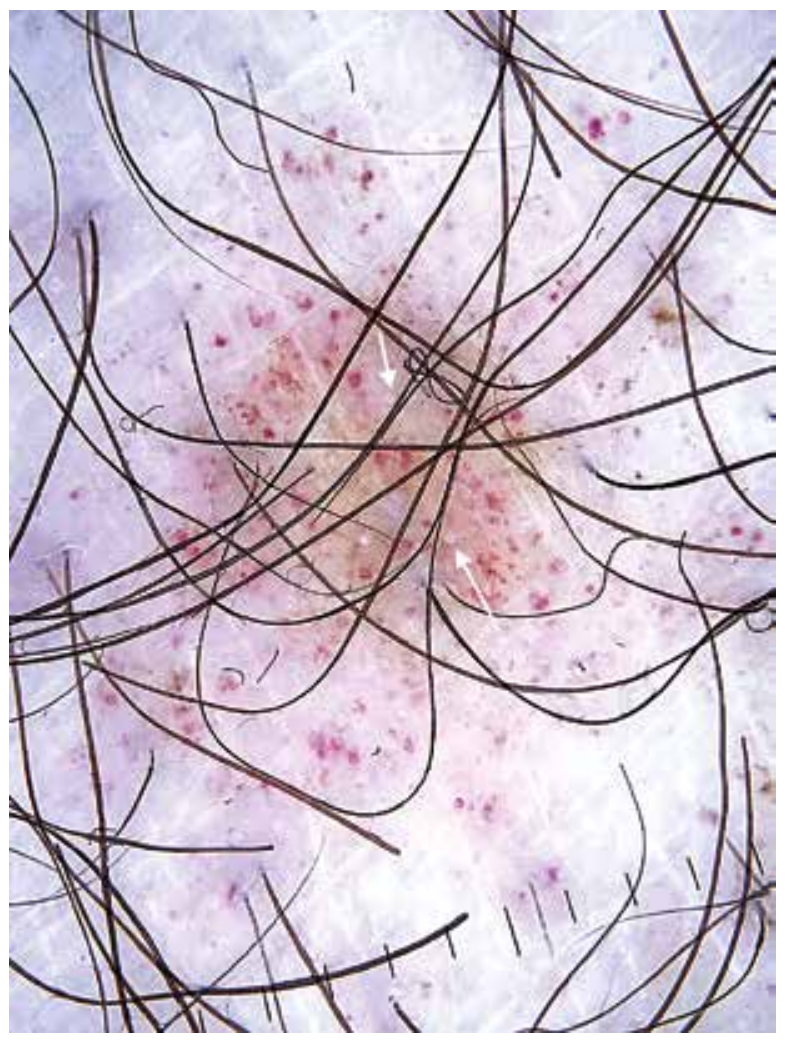

Figure 6. Rosette structures (arrows) 
nation of purpuric eruptions due to haemorrhagic diathesis shows homogenous structureless red areas; however, we thought that bleeding diathesis should be excluded in all the cases showing purpuric eruption [19].

Another differential diagnosis of PPD is leukocytoclastic vasculitis. Purpuric lesions in leukocytoclastic vasculitis is palpable unlike those seen in PPD. The presence of some symptoms like painful cutaneous lesions, fever and arthralgia also supports leukocytoclastic vasculitis [20]. PPD is typically asymptomatic [5].

Angioma serpiginosum shows well-circumscribed red to purple lagoons on dermoscopic examination in contrast to PPD [21].

\section{Conclusions}

The characteristic dermoscopic findings of the entity have been defined previously; however, we have identified some dermoscopic findings which have not been described for PPD: light brown background, red circles, brown circles, red background and rosette structures. Dermoscopic examination may facilitate the diagnostic process in PPD by reducing the need for histopathological examination.

\section{Conflict of interest}

The authors declare no conflict of interest.

\section{References}

1. Coulombe J, Jean SE, Hatami A, et al. Pigmented purpuric dermatosis: clinicopathologic characterization in a pediatric series. Pediatr Dermatol 2015; 32: 358-62.

2. Hanna S, Walsh N, D'Intino Y, et al. Mycosis fungoides presenting as pigmented purpuric dermatitis. Pediatr Dermatol 2006; 23: 350-4.

3. Cavalcante ML, Masuda PY, Brito FF, et al. Schamberg's disease: case report with therapeutic success by using colchicine. An Bras Dermatol 2017; 92: 246-8.

4. Portela PS, Melo DF, Ormiga P, et al. Dermoscopy of lichen aureus. An Bras Dermatol 2013; 88: 253-5.

5. Sardana K, Sarkar R, Sehgal VN. Pigmented purpuric dermatoses: an overview. Int I Dermatol 2004; 43: 482-8.

6. Çakmak SK, Kılıç A, Yorulmaz A, et al. Dermoscopic findings in patients with pigmented purpuric dermatoses. Acta Dermatovenerol Croat 2016; 24: 291-5.

7. Ozkaya DB, Emiroglu N, Su O, et al. Dermatoscopic findings of pigmented purpuric dermatosis. An Bras Dermatol 2016; 91: 584-7.

8. Suh KS, Park JB, Yang MH, et al. Diagnostic usefulness of dermoscopy in differentiating lichen aureus from nummular eczema. J Dermatol 2017; 44: 533-7.

9. Zaballos P, Puig S, Malvehy J. Dermoscopy of pigmented purpuric dermatoses (lichen aureus): a useful tool for clinical diagnosis. Arch Dermatol 2004; 140: 1290-1.

10. Tolaymat L, Hall MR. Dermatitis, pigmented purpuric. In: StatPearls [Internet]. Treasure Island (FL): StatPearls Publishing; 2018 Jan. Available from: https://www.ncbi.nlm.nih. gov/books/NBK519562/
11. Gönül M, Külcü Çakmak S, Ozcan N et al. Clinical and laboratory findings of pigmented purpuric dermatoses. Ann Dermatol 2014; 26: 610-4.

12. Sharma L, Gupta S. Clinicoepidemiological study of pigmented purpuric dermatoses. Indian Dermatol Online J 2012; 3: 17-20.

13. Engin B, Ozdemir M, Kaplan M, Mevlitoğlu I. Patch test results in patients with progressive pigmented purpuric dermatosis. J Eur Acad Dermatol Venereol 2009; 23: 209.

14. de Giorgi V, Stante M, Massi D, et al. Possible histopathologic correlates of dermoscopic features in pigmented melanocytic lesions identified by means of optical coherence tomography. Exp Dermatol 2005; 14: 56-9.

15. Arpaia N, Cassano N, Vena GA. Lessons on dermoscopy: pigment network in nonmelanocytic lesions. Dermatol Surg 2004; 30: 929-30.

16. Kittler H, Rosendahl C, Cameron A, Tschandl P. Dermatoscopy. An algorithmic method based on pattern analysis. 2011. Facultas Verlags and Buchhandels AG, Universitatsverlag, Austria.

17. Zaballos P, Salsench E, Puig S, Malvehy J. Dermoscopy of venous stasis dermatitis. Arch Dermatol 2006; 142: 1526.

18. Liebman TN, Rabinovitz HS, Dusza SW, Marghoob AA. White shiny structures: dermoscopic features revealed under polarized light. J Eur Acad Dermatol Venereol 2012; 26: 1493-7.

19. Vazquez-Lopez F, García-García B, Sanchez-Martin J, Argenziano G. Dermoscopic patterns of purpuric lesions. Arch Dermatol 2010; 146: 938.

20. Aounallah A, Arouss A, Ghariani N, et al. Cutaneous leukocytoclastic vasculitis: about 85 cases. Pan Afr Med J 2017; 26: 138.

21. Ghanadan A, Kamyab-Hesari K, Moslehi H, Abasi A. Dermoscopy of angioma serpiginosum: a case report. Int J Dermatol 2014; 53: 1505-7. 